TITLE:

\title{
Time-periodic finite-element method for hysteretic eddy-current analysis
}

$\operatorname{AUTHOR}(S)$ :

Matsuo, T; Shimasaki, M

CITATION:

Matsuo, T ... [et al]. Time-periodic finite-element method for hysteretic eddy-current analysis. IEEE TRANSACTIONS ON MAGNETICS 2002, 38(2): 549-552

ISSUE DATE:

2002-03

URL:

http://hdl.handle.net/2433/39996

\section{RIGHT:}

(c)2002 IEEE. Personal use of this material is permitted. However, permission to reprint/republish this material for advertising or promotional purposes or for creating new collective works for resale or redistribution to servers or lists, or to reuse any copyrighted component of this work in other works must be obtained from the IEEE. 


\section{Time-Periodic Finite-Element Method for Hysteretic Eddy-Current Analysis}

Tetsuji Matsuo, Member, IEEE, and Masaaki Shimasaki, Member, IEEE

\begin{abstract}
The time-periodic finite-element method is applied to hysteretic eddy-current analysis. A new method for determining the relaxation factor of Newton iteration is proposed for fast convergence to periodic solutions. The influence of hysteretic terms in the Jacobian matrix on the Newton iteration is discussed. Application examples show that the proposed method efficiently yields periodic solutions for both hysteretic and nonhysteretic eddy-current fields.
\end{abstract}

Index Terms-Eddy-current analysis, hysteresis, Newton method, relaxation factor, time-periodic FEM.

\section{INTRODUCTION}

$\mathbf{F}$ INITE-ELEMENT analysis often requires a large amount of computation time to find periodic eddy-current fields in electric machines with nonlinear inductive elements, because these elements prevent transient oscillations from decaying quickly. If the inductive elements have hysteretic characteristics, steady-state analysis becomes much more time-consuming because of the computational cost for representing the hysteretic characteristics.

The present paper applies the time-periodic finite element method (FEM) [1]-[3] to a fast steady-state analysis of hysteretic eddy-current fields. Using the Newton iteration, the timeperiodic FEM yields steady periodic fields directly without transient calculation. To stabilize the Newton iteration, a relaxation factor is often introduced in the time-periodic FEM [3]. The present paper proposes a new and simple method for determining the relaxation factor for fast convergence to periodic solutions.

The influence of the hysteretic element on the time-periodic FEM is also discussed in this paper. When the magnetic field $H$ is represented as the hysteretic function of the magnetic field density $B, H$ depends on not only the present value of $B$ but also on the past history of $B$ [4], [5]. This relation requires hysteretic terms to represent the effect of past history in the Jacobian matrix for the Newton iteration. The effect of these hysteretic terms on the Newton iteration in the time-periodic FEM, however, has not yet been discussed. The present paper describes the hysteretic terms and shows that hysteretic terms are necessary for the fast convergence of Newton iteration.

Manuscript received July 2, 2001; revised October 25, 2001. This work was supported in part by the Kansai Research Foundation for technology promotion.

The authors are with the Graduate School of Engineering, Kyoto University, Kyoto 606-8501, Japan (e-mail: tmatsuo@kuee.kyoto-u.ac.jp; simasaki@kuee.kyoto-u.ac.jp).

Publisher Item Identifier S 0018-9464(02)01258-X.

\section{2-D EdDy-CuRrent ANALYsis With Voltage Source}

The two-dimensional (2-D) eddy-current field given by (1) is analyzed.

$$
\frac{\partial H_{\mathrm{y}}\left(B_{\mathrm{x}}, B_{\mathrm{y}}\right)}{\partial x}-\frac{\partial H_{\mathrm{x}}\left(B_{\mathrm{x}}, B_{\mathrm{y}}\right)}{\partial y}=j_{0}(I)-\sigma \frac{\partial A}{\partial t}
$$

where $\vec{H}=\left(H_{\mathrm{x}}, H_{\mathrm{y}}\right)$ is the magnetic field that is a nonlinear function of the magnetic flux density $\vec{B}=\left(B_{\mathrm{x}}, B_{\mathrm{y}}\right) ; A$ is the magnetic vector potential; $\sigma$ is the electrical conductivity; and $j_{0}$ is the induced current density. The exciting current $I$ is determined by the electric-circuit equation (2).

$$
E \cos \omega t-\frac{\mathrm{d} \Psi}{\mathrm{d} t}-R I=0
$$

where $E$ is the amplitude of voltage source; $\omega$ is the angular frequency; and $R$ is the resistance. The flux interlinkage $\Psi$ of winding is given by

$$
\Psi=\frac{n_{\mathrm{c}} l_{z}}{S_{\mathrm{c}}} \iint_{S_{\mathrm{w}}} A a_{\mathrm{z}} \mathrm{d} x \mathrm{~d} y
$$

where $n_{\mathrm{c}}$ is the number of turns; $S_{\mathrm{c}}$ is the cross-sectional area of winding; $l_{\mathrm{Z}}$ is the thickness; $a_{\mathrm{Z}}(= \pm 1)$ is the winding direction; and $S_{\mathrm{w}}$ is the winding region.

The Galerkin FEM and the backward Euler time-difference scheme lead to (4) from (1) and (2) [2].

$$
\begin{aligned}
\boldsymbol{F}_{n} & =\left[\begin{array}{l}
\boldsymbol{K}\left(A\left(t_{n}\right)\right)+\boldsymbol{M} \frac{A\left(t_{n}\right)-\boldsymbol{A}\left(t_{n-1}\right)}{\Delta t}-\boldsymbol{J}\left(I\left(t_{n}\right)\right) \\
\frac{E \Delta t \cos \omega t_{n}-\Psi\left(t_{n}\right)+\Psi\left(t_{n-1}\right)-R I\left(t_{n}\right) \Delta t}{l_{\mathrm{z}}}
\end{array}\right] \\
& =\mathbf{0}
\end{aligned}
$$

where $\Delta t$ is the time-step and the subscripts of $t$ indicate the time-points; $\boldsymbol{K}=\left\{K_{i}\right\}, \boldsymbol{M}=\left\{M_{i j}\right\}, \boldsymbol{J}=\left\{J_{i}\right\}$ and $\boldsymbol{A}=$ $\left\{A_{i}\right\}$. These vectors and matrix are given by

$$
\begin{aligned}
K_{i} & =\sum_{e} \iint_{S e}\left(H_{\mathrm{x}} \frac{\partial N_{i}}{\partial y}-H_{\mathrm{y}} \frac{\partial N_{i}}{\partial x}\right) \mathrm{d} x \mathrm{~d} y \\
M_{i j} & =\sum_{e} \iint_{S e} \sigma N_{i} N_{j} \mathrm{~d} x \mathrm{~d} y \\
J_{i} & =\sum_{e} \iint_{S e} j_{0} N_{i} \mathrm{~d} x \mathrm{~d} y
\end{aligned}
$$

where $N_{i}$ is the interpolation function, $S_{e}$ is the region of element $e$, and $j_{0}$ is given in the winding region by $n_{\mathrm{c}} a_{\mathrm{z}} I / S_{\mathrm{c}}$. 


\section{TIME-PERIODIC FEM WithouT HySTERESIS}

We assume a symmetric periodic solution of (4) as

$$
\boldsymbol{X}\left(t_{0}\right)=-\boldsymbol{X}\left(t_{N}\right)
$$

where $X=(A, I), t_{N}=t_{0}+T / 2$, and $T=2 \pi / \omega$ is the time-period.

The time-periodic FEM yields the periodic solution from (4) and (8) using the Newton iteration

$$
\boldsymbol{X}\left(t_{n}\right)^{m+1}=\boldsymbol{X}\left(t_{n}\right)^{m}+\alpha \delta \boldsymbol{X}\left(t_{n}\right)
$$

where $\alpha$ is a relaxation factor, and $\delta X\left(t_{n}\right)(n=1, \ldots, N)$ are determined by the linear system of (10) and (11).

$$
\begin{aligned}
\boldsymbol{G}_{1} \delta \boldsymbol{X}\left(t_{1}\right)-\boldsymbol{C}_{1} \delta \boldsymbol{X}\left(t_{N}\right) & =-\boldsymbol{F}_{1} \\
\boldsymbol{G}_{n} \delta \boldsymbol{X}\left(t_{n}\right)+\boldsymbol{C}_{n} \delta \boldsymbol{X}\left(t_{n-1}\right) & =-\boldsymbol{F}_{n}(n=2, \ldots, N)
\end{aligned}
$$

where

$$
\begin{aligned}
& \boldsymbol{G}_{n}=\left[\begin{array}{cc}
\frac{\partial \boldsymbol{K}}{\partial A\left(t_{n}\right)}+\frac{\boldsymbol{M}}{\Delta t} & -\frac{\partial \boldsymbol{J}}{\partial I} \\
-\left(\frac{\partial \boldsymbol{J}}{\partial I}\right)^{\mathrm{T}} & -\frac{R \Delta t}{l z}
\end{array}\right] \\
& \boldsymbol{C}_{n}=\left[\begin{array}{cc}
-\frac{\boldsymbol{M}}{\Delta t} & 0 \\
\left(\frac{\partial \boldsymbol{J}}{\partial I}\right)^{\mathrm{T}} & 0
\end{array}\right] .
\end{aligned}
$$

The matrix $\partial \boldsymbol{K} / \partial A=\left\{\partial K_{i} / \partial A_{j}\right\}$ is given by

$$
\begin{aligned}
\frac{\partial K_{i}}{\partial A_{j}}=\sum_{e} & \iint_{S e}\left\{\left(\frac{\partial H_{\mathrm{x}}}{\partial B_{\mathrm{x}}} \frac{\partial N_{j}}{\partial y}-\frac{\partial H_{\mathrm{x}}}{\partial B_{\mathrm{y}}} \frac{\partial N_{j}}{\partial x}\right) \frac{\partial N_{i}}{\partial y}\right. \\
& \left.-\left(\frac{\partial H_{\mathrm{y}}}{\partial B_{\mathrm{x}}} \frac{\partial N_{j}}{\partial y}-\frac{\partial H_{\mathrm{y}}}{\partial B_{\mathrm{y}}} \frac{\partial N_{j}}{\partial x}\right) \frac{\partial N_{i}}{\partial x}\right\} \mathrm{d} x \mathrm{~d} y .
\end{aligned}
$$

Since the linear system of (10) and (11) may become very large, an approximation (14) is often used to solve (10) for $\delta \boldsymbol{X}\left(t_{1}\right)$ in the time-periodic FEM

$$
\delta \boldsymbol{X}\left(t_{N}\right)=\mathbf{0} .
$$

Then $\delta \boldsymbol{X}\left(t_{n}\right)(n=2, \ldots, N)$ are sequentially obtained from (11).

While $\delta \boldsymbol{X}\left(t_{n}\right)(n=1, \ldots, N)$ satisfy (11), they do not satisfy (10) owing to the approximation (14) in solving (10). This often causes the failure of Newton iteration (9) with $\alpha=1$. The present paper accordingly proposes that the relaxation factor $\alpha$ is determined so as to minimize

$$
\operatorname{Err}_{1}=\left\|\boldsymbol{F}_{1}+\alpha\left\{\boldsymbol{G}_{1} \delta \boldsymbol{X}\left(t_{1}\right)-\boldsymbol{C}_{1} \delta \boldsymbol{X}\left(t_{N}\right)\right\}\right\| .
$$

Since $G_{1} \delta \boldsymbol{X}\left(t_{1}\right)=-\boldsymbol{F}_{1}$ from (10) and (14), $\operatorname{Err}_{1}$ is given by

$$
\operatorname{Err}_{1}=\left\|(1-\alpha) \boldsymbol{F}_{1}-\alpha \boldsymbol{C}_{1} \delta \boldsymbol{X}\left(t_{N}\right)\right\|
$$

This is minimized by $\alpha=\alpha_{\mathrm{opt}}$ that is given by

$$
\alpha_{\mathrm{opt}}=\left[\boldsymbol{F}_{1} \cdot\left\{\boldsymbol{F}_{1}+\boldsymbol{C}_{1} \delta \boldsymbol{X}\left(t_{N}\right)\right\}\right] /\left\|\boldsymbol{F}_{1}+\boldsymbol{C}_{1} \delta \boldsymbol{X}\left(t_{N}\right)\right\|^{2} \text {. }
$$

\section{TIME-PERIODIC FEM WiTH HYSTERESIS}

When $\vec{H}$ is a hysteretic function of $\vec{B}, \vec{H}$ depends on not only the present value of $\vec{B}$ but also the past history of $\vec{B}$ [4], [5] as

$$
\vec{H}=\vec{H}_{n}\left(\vec{B}\left(t_{n}\right) \mid \vec{B}\left(t_{0}\right), \vec{B}\left(t_{1}\right), \ldots, \vec{B}\left(t_{n-1}\right)\right)
$$

In the case of symmetric periodic oscillation without accommodation, only the history of the past half-period affects the present field as

$$
\begin{array}{r}
\vec{H}=\vec{H}_{n}\left(\vec{B}\left(t_{n}\right) \mid-\vec{B}\left(t_{n+1}\right),-\vec{B}\left(t_{n+2}\right), \ldots,-\vec{B}\left(t_{N}\right),\right. \\
\left.\vec{B}\left(t_{1}\right), \vec{B}\left(t_{2}\right), \ldots, \vec{B}\left(t_{n-1}\right)\right) .
\end{array}
$$

The vector function $\boldsymbol{K}$ is accordingly written as

$$
\begin{array}{r}
\boldsymbol{K}=\boldsymbol{K}_{n}\left(\boldsymbol{A}\left(t_{n}\right) \mid-\boldsymbol{A}\left(t_{n+1}\right),-\boldsymbol{A}\left(t_{n+2}\right), \ldots,-\boldsymbol{A}\left(t_{N}\right)\right. \\
\left.\boldsymbol{A}\left(t_{1}\right), \boldsymbol{A}\left(t_{2}\right), \ldots, A\left(t_{n-1}\right)\right)
\end{array}
$$

Consequently, the linear system of (10) and (11) becomes

$$
\begin{gathered}
\boldsymbol{G}_{1} \delta \boldsymbol{X}\left(t_{1}\right)+\sum_{k \neq 1} \boldsymbol{G}_{h 1 k} \delta \boldsymbol{X}\left(t_{k}\right)-\boldsymbol{C}_{1} \delta \boldsymbol{X}\left(t_{N}\right)=-\boldsymbol{F}_{1} \\
\boldsymbol{G}_{n} \delta \boldsymbol{X}\left(t_{n}\right)+\sum_{k \neq n} \boldsymbol{G}_{\mathrm{h} n k} \delta \boldsymbol{X}\left(t_{k}\right)+\boldsymbol{C}_{n} \delta \boldsymbol{X}\left(t_{n-1}\right)=-\boldsymbol{F}_{n} \\
(n=2, \ldots, N)
\end{gathered}
$$

$$
\boldsymbol{G}_{\mathrm{h} n k}=\left[\begin{array}{cc}
\partial \boldsymbol{K}_{n} / \partial A\left(t_{k}\right) & \mathbf{0} \\
\mathbf{0} & 0
\end{array}\right]
$$

where $\boldsymbol{G}_{\mathbf{h} n k}$ represents the hysteretic effect in the Jacobian matrix since the summation terms in (21) and (22) are caused by the hysteretic relations (19) and (20).

The matrix $\partial \boldsymbol{K}_{n} / \partial \boldsymbol{A}\left(t_{k}\right)=\left\{\partial K_{n i} / \partial A_{j}\left(t_{k}\right)\right\}$ is given by

$$
\begin{aligned}
& \partial K_{n i} / \partial A_{j}\left(t_{k}\right) \\
& =\sum_{e} \iint_{S e}\left\{\left(\frac{\partial H_{\mathrm{x} n}}{\partial B_{\mathrm{x}}\left(t_{k}\right)} \frac{\partial N_{j}}{\partial y}-\frac{\partial H_{\mathrm{x} n}}{\partial B_{\mathrm{y}}\left(t_{k}\right)} \frac{\partial N_{j}}{\partial x}\right) \frac{\partial N_{i}}{\partial y}\right. \\
& \left.\quad-\left(\frac{\partial H_{\mathrm{y} n}}{\partial B_{\mathrm{x}}\left(t_{k}\right)} \frac{\partial N_{j}}{\partial y}-\frac{\partial H_{\mathrm{y} n}}{\partial B_{\mathrm{y}}\left(t_{k}\right)} \frac{\partial N_{j}}{\partial x}\right) \frac{\partial N_{i}}{\partial x}\right\} \mathrm{d} x \mathrm{~d} y
\end{aligned}
$$

where $\left\{K_{n i}\right\}=\boldsymbol{K}_{n}$ given by $(20)$, and $\left(H_{\mathrm{x} n}, H_{\mathrm{y} n}\right)=\vec{H}_{n}$ given by (19).

Since the linear system of (21) and (22) may become very large, an approximation (25) is used to solve (21) for $\delta \boldsymbol{X}\left(t_{1}\right)$

$$
\delta \boldsymbol{X}\left(t_{k}\right)=\mathbf{0}(k=2, \ldots, N)
$$

Then, (22) is sequentially solved for $\delta \boldsymbol{X}\left(t_{n}\right)(n=2, \ldots, N)$ with the approximation (26)

$$
\delta \boldsymbol{X}\left(t_{k}\right)=\mathbf{0}(k=n+1, \ldots, N) .
$$




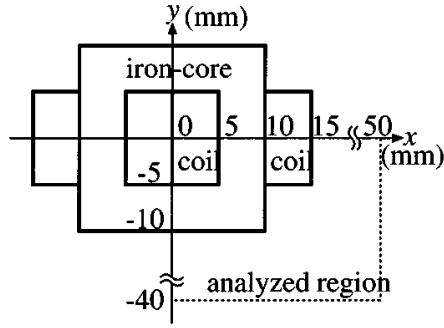

Fig. 1. Analyzed iron-cored inductor.

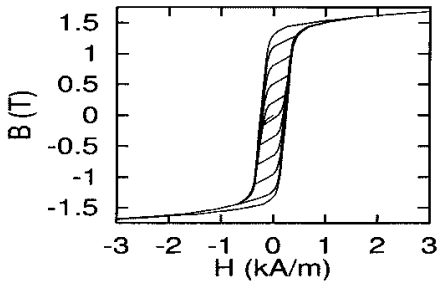

Fig. 2. Hysteretic characteristics.

In a way similar to the nonhysteretic case, the relaxation factor $\alpha$ is determined so as to minimize

$$
\begin{array}{r}
\operatorname{Err}_{1}=\| \boldsymbol{F}_{1}+\alpha\left\{\boldsymbol{G}_{1} \delta \boldsymbol{X}\left(t_{1}\right)+\sum_{k=2}^{N} \boldsymbol{G}_{\mathrm{h} 1 k} \delta \boldsymbol{X}\left(t_{k}\right)\right. \\
\left.-\boldsymbol{C}_{1} \delta \boldsymbol{X}\left(t_{N}\right)\right\} \| \\
=\|(1-\alpha) \boldsymbol{F}_{1}+\alpha\left\{\sum_{k=2}^{N} \boldsymbol{G}_{\mathrm{h} 1 k} \delta \boldsymbol{X}\left(t_{k}\right)\right. \\
\left.-\boldsymbol{C}_{1} \delta \boldsymbol{X}\left(t_{N}\right)\right\} \| .
\end{array}
$$

This reaches the minimum when $\alpha=\alpha_{\mathrm{opt}}$ given by

$$
\alpha_{\mathrm{opt}}=\frac{\boldsymbol{F}_{1} \cdot\left\{\boldsymbol{F}_{1}+\boldsymbol{C}_{1} \delta \boldsymbol{X}\left(t_{N}\right)-\sum_{k=2}^{N} \boldsymbol{G}_{\mathrm{h} 1 k} \delta \boldsymbol{X}\left(t_{k}\right)\right\}}{\left\|\boldsymbol{F}_{1}+C_{1} \delta \boldsymbol{X}\left(t_{N}\right)-\sum_{k=2}^{N} \boldsymbol{G}_{\mathrm{h} 1 k} \delta \boldsymbol{X}\left(t_{k}\right)\right\|^{2}} .
$$

\section{EXAMPLES OF EDDY-CURRENT ANALYSIS}

The iron-cored inductor shown in Fig. 1 is analyzed. The circuit parameters are set as $E / l_{\mathrm{z}}=500 \mathrm{~V} / \mathrm{m}, \omega=100 \pi$ $\mathrm{rad} / \mathrm{s}, n_{\mathrm{c}}=400$ and $R / l_{\mathrm{z}}=50 \Omega / \mathrm{m}$. The electrical conductivity $\sigma$ is set at $1 \times 10^{6} \mathrm{~S} / \mathrm{m}$. The present analysis assumes an isotropic vector hysteresis. Fig. 2 shows the unidirectional hysteretic characteristics of the iron-core constructed by the superposition of stop hysteron models [6]. This model can analytically provide $\partial \vec{H}_{n} / \partial \vec{B}\left(t_{k}\right)$ required in (24). The analyzed region shown in Fig. 1 is divided into 6000 triangular meshes, where the iron-core is divided into 600 equal meshes. The time-step $\Delta t$ is set at $\pi /(12 \omega)$. The computation has been

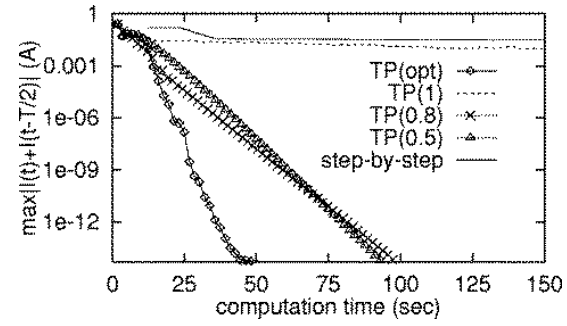

(a)

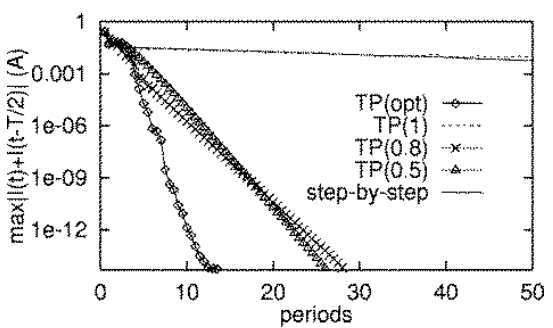

(b)

Fig. 3. Convergence of exciting current of nonhysteretic inductor; (a) required periods, and (b) required CPU time.

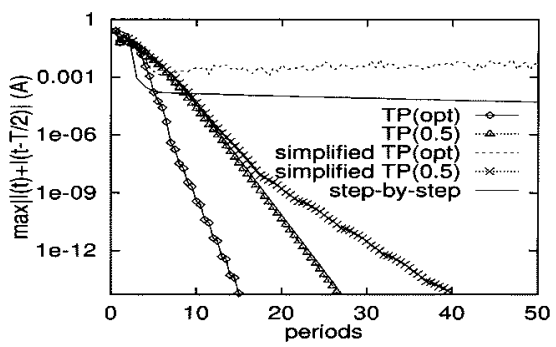

(a)

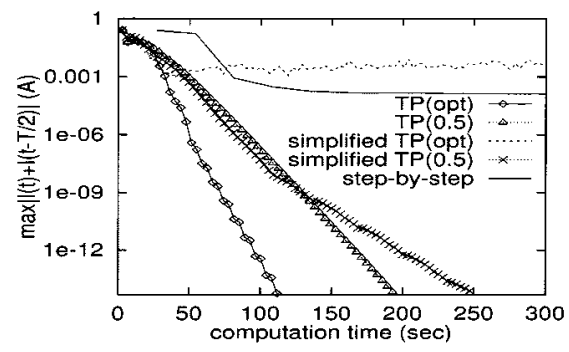

(b)

Fig. 4. Convergence of exciting current of hysteretic inductor; (a) required periods, and (b) required CPU time.

performed on a PC using the Intel Pentium II (450 MHz) processor.

First, the time-periodic FEM is compared with a conventional step-by-step method for eddy-current analysis without hysteresis, where the initial curve of the hysteretic characteristics shown in Fig. 2 is used for the nonhysteretic magnetic characteristics. Fig. 3 shows the convergence of exciting current where the maximum value of $|I(t)+I(t-T / 2)|$ during half-period is plotted. The initial guess $X\left(t_{n}\right)^{0}(n=1, \ldots, N)$ are set at 0 for the time-periodic FEM, and the initial condition $X\left(t_{0}\right)$ is set at $\mathbf{0}$ for the step-by-step method at $t_{0}=0$. One Newton iteration in the time-periodic FEM corresponds to a half-period in Fig. 3(a). The curve "TP(opt)" shows the convergence given by the time-periodic FEM with a relaxation factor $\alpha=0.5$ for the first five Newton iterations and $\alpha_{\mathrm{opt}}$ given by (17) for 


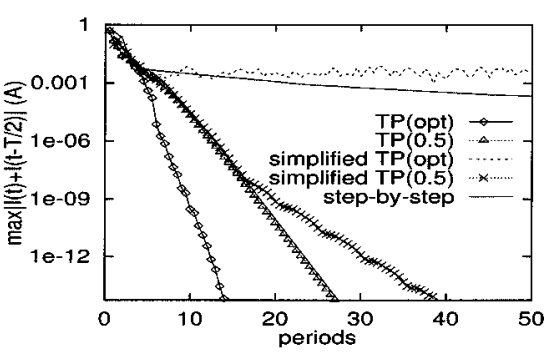

(a)

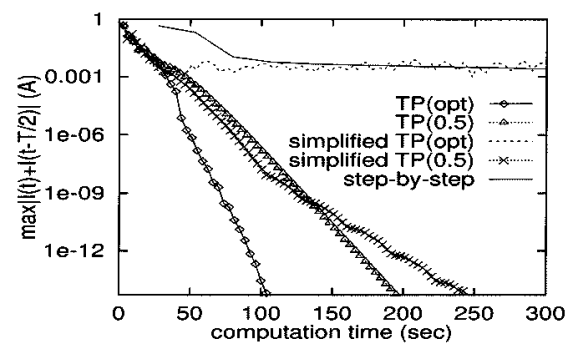

(b)

Fig. 5. Convergence of exciting current of hysteretic inductor driven by voltage source $E \sin \omega t$; (a) required periods, and (b) required CPU time.

the rest of the iterations. The curves "TP(1)" and "TP(0.5)" are given by the time-periodic FEM with $\alpha=1$ and 0.5 , respectively. Fig. 3 shows that the time-periodic FEM with the proposed relaxation factor achieves much faster convergence than does the step-by-step method, whereas the time-periodic FEM with $\alpha=1$ results in the failure of convergence.

Next, the hysteretic eddy-current field is analyzed. Fig. 4 shows the convergence of the exciting current of the hysteretic inductor. The "simplified-TP" curves mean that the hysteretic terms with $G_{\mathrm{h} m k}$ in (21), (22), and (28) are neglected for the sake of simplicity in the same way as the nonhysteretic case. The convergence of the step-by-step method is shown by $\max |I(t)-I(t-T)|$ because the step-by-step method does not always yield a symmetric periodic field that satisfies (8). Fig. 4(a) shows that the simplified method results in the failure or slow convergence of Newton iteration because of the neglect of hysteretic terms in the Jacobian matrix. Fig. 4(b) shows that the step-by-step method consumes much more computation time for convergence because the step-by-step method requires several Newton iterations at every time-step, leading to a larger computational cost for one period than does the time-periodic FEM.

Fig. 5 shows the convergence of the exciting current of the hysteretic inductor that is driven by the voltage source of $E \sin (\omega t)$ instead of $E \cos (\omega t)$ in (2) and (4). Fig. 5 shows that the step-by-step method results in slower convergence than does the case driven by $E \cos (\omega t)$. This means that the step-by-step method may need a large number of periods when the initial values much differ from the steady solution. In contrast, the time-periodic FEM yields the steady solution as fast as shown in Fig. 4.

Fig. 6 shows the variation of relaxation factor corresponding to "TP(opt)" in Figs. 3-5.

Fig. 7 shows the steady $H_{\mathrm{x}}-B_{\mathrm{X}}$ loop given by the time-periodic FEM, and the $H_{\mathrm{x}}-B_{\mathrm{x}}$ trajectories given by the step-by-step method at $(x, y)=(0 \mathrm{~mm},-5 \mathrm{~mm})$. Fig. 7 shows that the

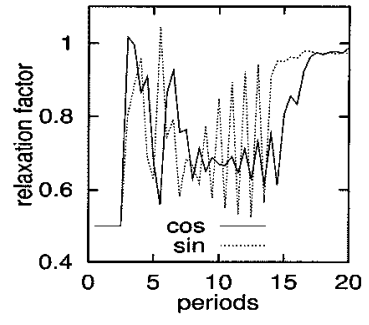

Fig. 6. Variation of relaxation factor.

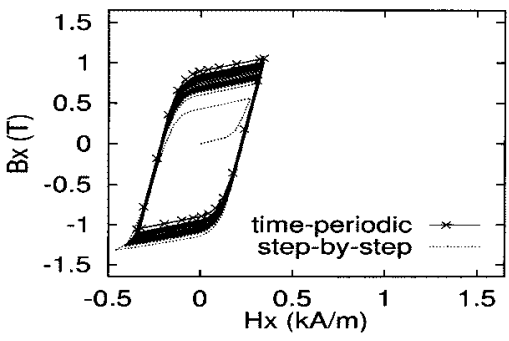

(a)

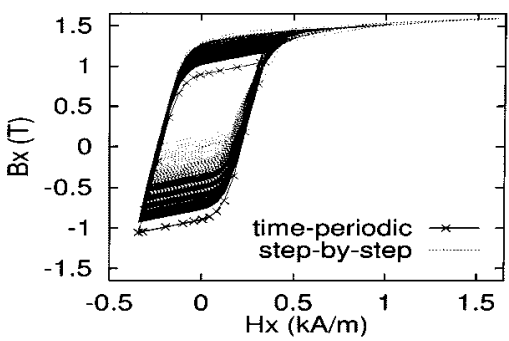

(b)

Fig. 7. Hysteresis loops; (a) driven by $E \cos \omega t$, and (b) driven by $E \sin \omega t$.

step-by-step method results in asymmetric steady loops whose positions depend on their past histories until arriving at the steady-state, whereas the time-periodic FEM yields a symmetric steady loop by the assumption of (8).

\section{CONCLUSION}

The proposed method efficiently yields periodic solutions for both hysteretic and nonhysteretic eddy-current fields. The neglect of hysteretic terms in the Jacobian matrix causes the failure or slow convergence of the Newton iteration.

\section{REFERENCES}

[1] T. Hara, T. Naito, and J. Umoto, "Field analysis of corona shield region in high voltage rotating machines by time-periodic finite element method," J. IEE Japan, vol. 102-B, pp. 423-430, July 1982.

[2] T. Nakata, N. Takahashi, K. Fujiwara, and A. Ahagon, "3-D nonlinear eddy-current analysis using the time-periodic finite element method," IEEE Trans. Magn., vol. 25, pp. 4150-4152, Sept. 1989.

[3] K. Muramatsu, N. Takahashi, T. Nakata, M. Nakano, Y. Ejiri, and J. Takehara, "3-D time-periodic finite element analysis of magnetic field in nonoriented materials taking into account hysteresis characteristics," IEEE Trans. Magn., vol. 33, pp. 1584-1587, Mar. 1997.

[4] T. Matsuo, K. Okumura, and A. Kishima, "A method for steady-state analysis of nonlinear systems with hysteretic elements," Jpn. Trans. Inst. Electron. Inf. Commun. Eng. A, vol. J73-A, pp. 478-485, Mar. 1990.

[5] — "Analysis of a hysteretic circuit containing an iron-cored inductor and a semiconductor switch," IEE Proc.-Circuits, Devices, Syst., vol. 146, pp. 176-183, Aug. 1999.

[6] T. Matsuo, Y. Osaka, and M. Shimasaki, "Eddy-current analysis using vector hysteresis models with play and stop hysterons," IEEE Trans. Magn., vol. 36, pp. 1172-1177, July 2000. 